“ (C) 2018 IEEE. Personal use of this material is permitted. Permission from IEEE must be obtained for all other uses, in any current or future media, including

reprinting/republishing this material for advertising or promotional purposes, creating new collective works, for resale or redistribution to servers or lists, or reuse of any copyrighted component of this work in other works." 


\title{
Time Series Analysis for Encrypted Traffic Classification: A Deep Learning Approach
}

\author{
Ly $\mathrm{Vu}^{1}$, Hoang V. Thuy ${ }^{1}$, Quang Uy Nguyen ${ }^{1}$, Tran N. Ngoc ${ }^{1}$, \\ Diep N. Nguyen ${ }^{2}$, Dinh Thai Hoang ${ }^{2}$, Eryk Dutkiewicz ${ }^{2}$ \\ ${ }^{1}$ Faculty of Information Technology, Le Quy Don Technical University, Hanoi, Vietnam \\ ${ }^{2}$ School of Electrical and Data Engineering, University of Technology Sydney, Australia
}

\begin{abstract}
We develop a novel time series feature extraction technique to address the encrypted application classification problem. The proposed method consists of two main steps. First, we propose a feature engineering technique to extract significant attributes of the encrypted network traffic behavior through analyzing the time series of receiving packets. In the second step, a deep learning technique is developed to exploit the advantage of time series data samples in providing the strong representation of the encrypted network applications. To evaluate the efficiency of the proposed solution on the encrypted application traffic classification problem, we carry out intensive experiments on a raw network traffic dataset, namely VPN-nonVPN, with three conventional classifier metrics including Precision, Recall, and F1 score. The experimental results demonstrate that our proposed approach can significantly improve the performance in identifying encrypted application traffic in terms of accuracy and computation efficiency.
\end{abstract}

Keywords- Traffic classification, LSTM, encrypted applications, deep learning, and feature engineering.

\section{INTRODUCTION}

Traffic classification is crucial to network resource management and security, e.g., policing, firewall, filtering, anomaly, and intrusion detection [1], [2]. To protect users' privacy (e.g., Viber, Whatsapp), most of applications come with an option to encrypt users' traffic. Moreover, malicious users also want to hide their behaviors through encrypted or covert tunnels [3]. As such traffic identification of encrypted applications plays a more and more pivotal role in cyber security.

Dealing with with encrypted traffic also brings many challenges for existing network traffic classifiers. Port-based techniques were one of the most popular methods used in the last decade to classify network traffic. However, they don't work well on many systems nowadays due to the development of dynamic and non-standard port applications. Instead of relying on port numbers, the payload-based and flow-based techniques that employ machine learning algorithms for network traffic identification can be adopted. However, encryption algorithms try to embroil data structure of packets, leading to reducing the effects of payload-based methods [4]. Alternatively, the flowbased methods usually exploit statistical features of network flows [5], [6], [7], resulting in increasing the computation resource and decreasing accuracy comparing with payloadbased methods. Therefore, conventional payload-based and flow-based techniques are not effective in both computation efficiency and representing encrypted packets for classification purpose.
Although many machine learning-based classification methods have been introduced to overcome the current limitations of conventional methods, their effectiveness strongly depends on the accuracy and effectiveness of the feature extraction process. However, as aforementioned, traffic of most applications has recently been encrypted [12], making the feature extraction from the raw traffic extremely challenging.

In this paper, we propose a novel deep learning-based classification approach using an effective feature engineering technique to represent encrypted network traffic. First, we rely on the time dependency of packets (i.e., time series samples) to better represent the behavior of traffic. We then leverage the advantage of Long Short-Term Memory (LSTM) network [4] [8] to extract the time dependency from the encrypted traffic. To verify the efficiency of the proposed solution, we deploy experiments on the real packet dataset, i.e., VPN-nonVPN dataset [13], with 12 kinds of applications. The experimental results show the efficiency of the our proposed approach in terms of accuracy, reliability, and robustness.

The main contributions of the paper are as follows:

- We propose a feature engineering technique that combines the payload-based method and flow-based to efficiently represent the behavior of encrypted network traffic.

- We develop a deep learning approach using LSTM to retain time dependency of receiving network traffic through time series analysis for data of network application traffic.

- We perform experiments to demonstrate the impact of the feature engineering technique to determine the best feature set that achieves the high accuracy with low computation requirement for identifying encrypted application traffic.

The rest of paper is organized as follows. Related works in the network traffic classification with unencrypted and encrypted traffic are discussed in Section II. Section III presents the fundamental background of the LSTM model. Our feature engineering technique is then described in Section IV. The testing dataset and experimental settings are in Section refexp. Section VI presents experiment results. Conclusion and future works are discussed in Section VII.

\section{RELATED WORK}

Flow-based, packet-based, and flow-packet combination are three major techniques to analyze network traffic in the literature. 
1) Flow-based method: A flow is a tuple of packets which have same source Internet Protocol (IP) address, destination IP address, source transport layer port, destination transport layer port, and transport protocol. Previous works [5], [6], [7] showed the effectiveness of the flow-based method for intrusion detection systems in classifying malicious traffic from normal traffic. Accordingly, this method is efficient in analyzing the behaviors of the set of packets. Gil et al. [14] introduced time-based attributes of packets for traffic analysis. However, these attributes need high volume of storage to store packets over a period of time [7]. As a result, the collection of many packets into a flow before extracting features is timeconsuming.

2) Payload-based method: Payload-based method has a high processing speed because it processes one packet at a time. However, this method is ineffective in presenting user behaviors which are crucial in classifying network traffic. Analyzing encrypted packet payload without decrypting has received more attentions recently [15], [16]. Sherry et al. [15] only considered the HTTP protocol with TLS encryption which is one of many encryption protocols on the Internet applications. S. Leroux et al. [16] presented a network traffic analysis method based on packet size and interval time. However, it only works efficiently for some specific applications which have extremely different packet size and transmission time, e.g., HTTP, VoIP, Video streaming, and P2P. Deep learning was also introduced for feature extraction of raw packets [4]. However, the method in [4] extracts entire raw packets to put into deep learning networks. This method can work for unencrypted traffic, but it is inefficient with encrypted traffic (detailed discussion in Section III).

3) Payload-flow based combination method: The payloadflow based combination method which consists of both packet and flow features is widely used in many well-known benchmark dataset, e.g., UNSW-NB15 [9] and NSL-KDD [10]. This method extracts features of packets combining with features of flow, leading to be able to present characteristics of traffic behaviors rapidly. Thus, this method is appropriate for encrypted network traffic because they can extract unencrypted packet headers along with encrypted payload. Furthermore, this method can use the flow-based technique to represent network traffic behaviors accurately. As a result, the packetflow based combination method is widely implemented in intrusion detection systems to identify network malicious traffic [11].

There are also some research works using the deep learning approach such as Stack Auto Encoder (SAE), CNN, LSTM network [4] [8] for classifying network traffic. However, these works only focus on the structure of deep network model to extract a large number of payload bytes from raw packets with large size of the flow. Consequently, the deep network requires a extremely large number of hidden layers with many neurons to enhance its accuracy. The work of Zhang et al. [2] showed the effectiveness of the time series analysis of network traffic for the network traffic classification problem. They extracted the hand-craft statistical features of packets and flow to represent traffic application, leading to heavy dependence of accuracy on human knowledge and high computation re-

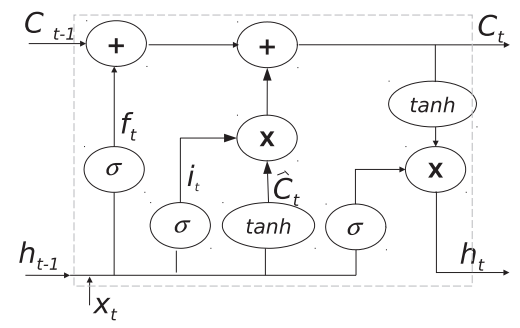

Fig. 1: The description of LSTM node.

source [1]. In this paper, we only extract packet features which strongly represent encrypted packets according to applications and arrange receiving packet samples in a time-series order. Furthermore, we take advantage from LSTM network in order to extract the time dependence of time-series packets automatically. This will not only retain the characteristic of traffic efficiently, but also perform accurately and rapidly in classifying encrypted network traffic applications.

\section{LONG Short Term Memory}

In this section, we describe a specific type of Recurrent Neuron Networks (RNN) [17], i.e., Long Short Term Memory (LSTM). LSTM is designed to avoid long-term dependency problem which cannot be resolved in RNN. This network structure was originally introduced by Hochreiter et al. [18], and has been refined as a powerful technique to handle the problem of time series prediction [19]. The difference of LSTM comparing with RNN resides in their nodes or cells. The basic structure of cell is presented in Fig. 1. One of the advantages of the LSTM is its ability to remove or add information to the cell state by a gate structure. There are three gates in each cell, i.e., input gate, forget gate and output gate, as shown in Fig. 1. Based on the strength of the information which is received by each node, each node will decide to block or pass the information. The calculations of three gates are briefly shown in equations (1)-(6).

$$
\begin{gathered}
f_{t}=\sigma\left(W_{f} \cdot\left[h_{t-1}, x_{t}\right]+b_{f}\right) . \\
i_{t}=\sigma\left(W_{i} \cdot\left[h_{t-1}, x_{t}\right]+b_{i}\right) . \\
\hat{C}_{t}=\tanh \left(W_{C} \cdot\left[h_{t-1}, x_{t}\right]+b_{C}\right) . \\
C_{t}=f_{t} C_{t-1}+i_{t} \hat{C}_{t} . \\
o_{t}=\sigma\left(W_{o} \cdot\left[h_{t-1}, x_{t}\right]+b_{o}\right) . \\
h_{t}=o_{t} \tanh \left(C_{t}\right) .
\end{gathered}
$$

Firstly, the forget gate is used to decide what information from the previous cell would be passed to this cell. This gate uses a sigmoid function with input of $h_{t-1}$ presenting the output of the previous cell and $x_{t}$ presenting the input of the current cell and output $f_{t}$ as in (1). The cell will forget this 


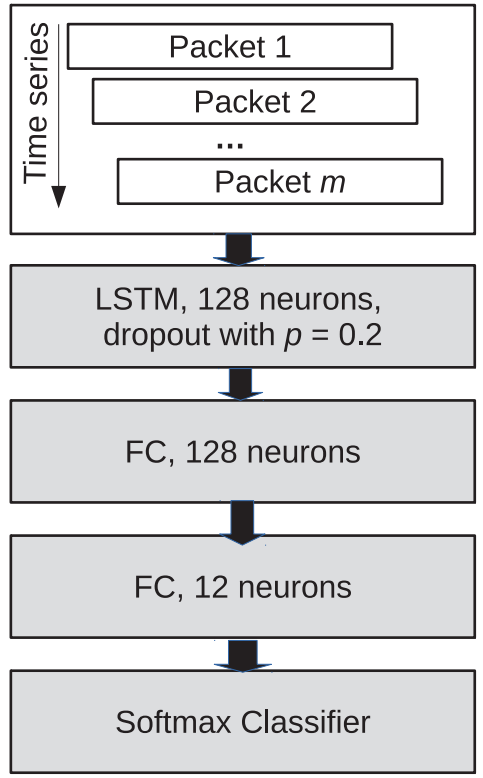

Fig. 2: The network architecture of our model.

information if $f_{t}$ is zero and remember otherwise. Secondly, the input gate decides which values will be updated in this cell. As presented in the Fig. 1, the sigmoid layer will decide values which will be updated, i.e., $i_{t}$ calculated in (2), and the tanh layer creates a vector of new candidate values, i.e., $\hat{C}_{t}$ calculated in (3). The state of the cell is computed based on output of the forget gate and the input gate which is described in (4). Finally, the cell has to decide the output value by the output gate. The inputs $h_{t-1}$ and $x_{t}$ pass though sigmoid layer to decide which parts of cell state being output as $o_{t}$ presented in (5). The Output gate is the combination of $o_{t}$ and the tanh layer of cell state $C_{t}$ as shown in (6). If the time sequence length is $m$, the final output will be $h_{m}$ which is the value of $h_{t}$ when $t=m$.

One hidden layer has many LSTM cells as described above and one deep learning model is usually the combination of many hidden layers. By computing the partial derivatives of outputs, weights, and input values of hidden layers, the system can move backward to trace the error between real output values and predicted output values, and uses Gradient Descent method to update the weights concurrent in order to reduce the predicted errors. Therefore, the LSTM cell is able to use back propagation through the time. Furthermore, the LSTM cell considers one sample based on two characteristics, i.e., the value and the position in time series of the sample. This means that two input samples at different times may have the same values; however, the output will likely differ. Accordingly, the LSTM network can understand the context of samples better.

The network structure of our work is presented in Fig. 2 which includes three hidden layers: LSTM (128 neurons), two Fully Connected (FC) layers with 128 and 12 neurons, respectively. The softmax classifier layer with 12 neurons (according to 12 network applications in the dataset) is used as the last layer to classify input data. As shown in Fig. 2, the input data sample is the time sequence data, i.e., the network flow, which has size of $m$ packets.

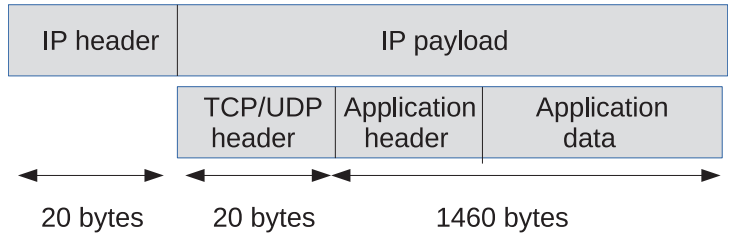

Fig. 3: The packet structure.

\section{Feature engineering Method}

In this section, we first analyze characteristics of an encrypted network packet, then propose the feature set that can represent encrypted network packets accurately.

Packet is data unit of the network layer in the Transmission Control Protocol/ Internet Protocol (TCP/IP) model. Most of previous works use entire payload of packets as features such as 1500 bytes [4] and 1000 bytes [8]. Fig. 3 shows that the packet includes 20 bytes of IP header, 20 bytes of transport layer header, and all the rest of bytes for application header and application data. In ubiquitous encrypted applications, the application layer data is encrypted using symmetric encryption algorithms to ensure the speed of the encryption and decryption process such as AES256 and RC4 [20]. In other words, the encryption keys are pseudo-random numbers which are changed in different sessions [20]. Therefore, the values of encrypted application layer data are completely different between sessions, although they belong to one type of application. As a result, the application layer data is unable to represent the application type. However, the header of application layer describes the encryption algorithm names and protocols which are significant for identifying network traffic.

Fig. 4 shows features which we extract from IP packets. In this figure, features from 1 to 3 describe the identification of a flow including source transport layer port, destination transport layer port, and protocol. The packet samples are ordered in a time series, so we don't need IP address in collecting the flow. In this work, we use these information to recognize the flow of continuous packets. The fourth feature presents the size of application data which strongly represents the difference between network traffic applications. Features from 5 to 44 are exactly the byte values of the IP header and transport header which are not encrypted in encrypted IP packets. For transport layer header, there are two popular protocols, i.e., User Datagram Protocol (UDP) and Transmission Control Protocol (TCP). The size of UDP header is 8 bytes, while the size of TCP header is usually 20 bytes. Therefore, we pad zero bytes at the end of UDP header to achieve the header size of 20 bytes. The rest of features are the first $n$ bytes of the application which present application layer data. In the experiment, we conduct the feature sets of data with various values of $n$, i.e., 10, 30, 50, 100, 200, 500, 1000, and 1500 . Packets with application layer data smaller than $n$ are padded by zero bytes to ensure the feature length.

\section{Experimental Settings and Evaluation Metrics}

This section presents the dataset and evaluation metrics which we use in the experiments. 


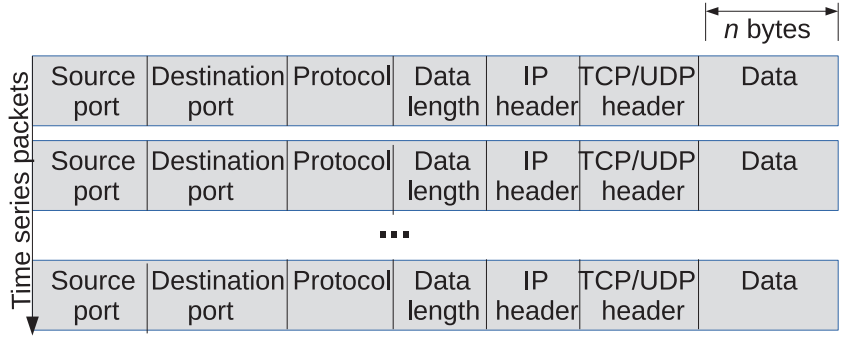

Fig. 4: The description of feature extraction.

TABLE I: Number of class samples for training and testing set

\begin{tabular}{||c|c|c||}
\hline Class & Training set & Testing set \\
\hline Chat & 948 & 270 \\
\hline Email & 328 & 76 \\
\hline File transfer & 739 & 195 \\
\hline P2P & 1946 & 482 \\
\hline Streaming & 2304 & 563 \\
\hline VoIP & 495 & 112 \\
\hline VPN-Chat & 1807 & 476 \\
\hline VPN-Email & 372 & 91 \\
\hline VPN-File transfer & 1306 & 304 \\
\hline VPN-P2P & 191 & 50 \\
\hline VPN-Streaming & 657 & 155 \\
\hline VPN-VoIP & 299 & 74 \\
\hline \hline Sequence total & $\mathbf{1 1 3 9 2}$ & $\mathbf{2 8 4 8}$ \\
\hline
\end{tabular}

\section{A. Dataset}

In order to test the effectiveness of the proposed method, we use well-known encrypted network traffic datasets, i.e., VPNnonVPN [13], which was created by the Canadian Institute for Cybersecurity. To generate the dataset, they created accounts for two users, i.e., Bob and Alice, who execute network transmissions to generate traffic for applications. The captured pcap files are labeled by applications which they engaged to. There are 12 distinct labels shown in Table I. To compare with other works on VPN-nonVPN dataset, we divide the dataset into training set $(80 \%)$ and testing set (20\%), yielding to the number of each class sample displayed in Table I. For each training epoch, $10 \%$ number of samples are chosen for validating the best model.

\section{B. Evaluation Metrics}

We use three popular evaluation metrics in the classification problem to assess the impact of our proposed method. The reported evaluation metrics include Precision score, Recall score, and F1-score [21]. These metrics are calculated for each class $i$ in the dataset by considering the one with all classifiers. Equations (7) and (8) present precision and recall score for one class. The final values of metrics are the weighted averages of all classes based on the sample size of each class in the testing set.

$$
\begin{gathered}
\text { Precision }=\frac{T P}{T P+F P} . \\
\text { Recall }=\frac{T P}{T P+F N} .
\end{gathered}
$$

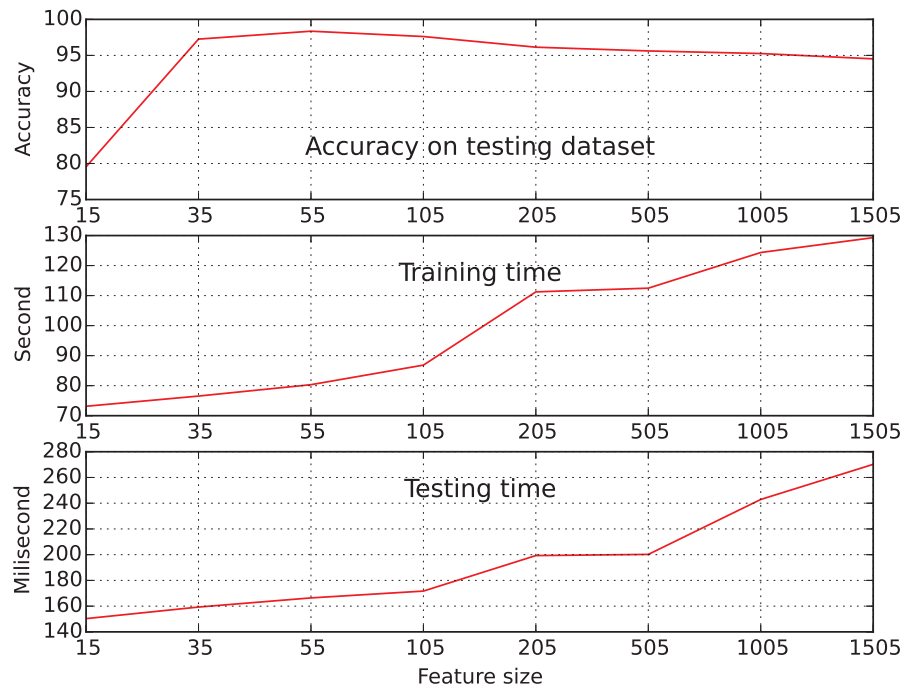

Fig. 5: Impact of feature size on accuracy and processing time.

The precision and recall score are calculated as in Equations (7) and (8), where $T P$ and $F P$ are the number of correct and incorrect predicted samples for a class $i$ respectively, and $F N$ is the number of incorrect predicted samples of the rest of classes. The advantage of these metrics is that they are very intuitive and easy to implement. However, they make no distinction between classes, that is sometime insufficient to measure a classifier, especially for imbalanced datasets.

The F1-score overcomes the disadvantage of the precision and recall score. F1 score is Harmonic mean [21] of Precision and Recall, where Harmonic mean is an appropriate way to average ratios. Therefore, it is not effected by the difference of the size of classes. Precisely, F1 score is computed in Equation (9). The F1-score is often considered as a reliable metric to evaluate the performance of classification algorithms in many kinds of dataset. Thus, we will use this metric for measuring our model and compare with other works in the next section. Precision and Recall will be presented as references.

$$
F 1=2 \times \frac{\text { Precision } \times \text { Recall }}{\text { Precision }+ \text { Recall }} .
$$

\section{RESUlTS AND Discussion}

In this section, we conduct two kinds of experiments to measure the impacts of our feature engineering technique and the accuracy of our proposed model.

\section{A. Impacts of Feature Engineering Technique}

In these experiments, we analyze the impacts of the feature engineering technique to the accuracy of the deep learning model. Fig. 5 presents the effects of feature size on classification accuracy and processing time. In this figure, the training time measures the processing time to execute 50 epochs in seconds and the testing time represents the processing time to predict all samples in the testing dataset. As shown in Fig. 5, the training time and predicting time increase along 


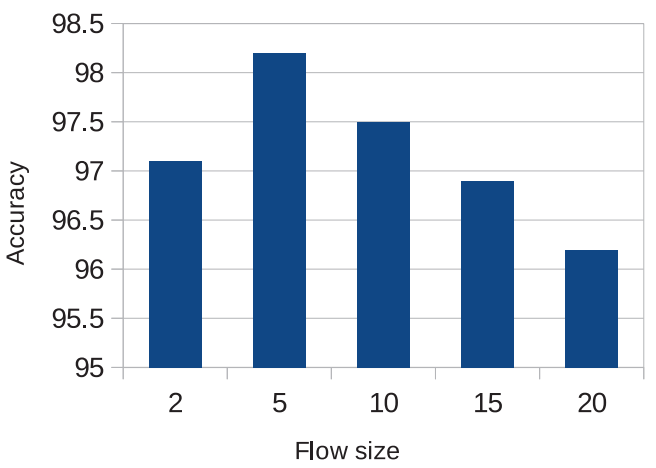

Fig. 6: Impact of flow size on accuracy.

with the expansion of the feature size. This is reasonable for executing a deep learning model because larger feature size always requires higher computation resource and more time consuming. In addition, Fig. 5 shows that the feature size 55 which contains 50 bytes of application layer data is the most effective to present encrypted network applications. As mentioned in Section IV, each network transaction is encrypted with a different key; therefore, using the large number of encrypted payload reduces the ability to represent network applications. Hence, we consider simulations on the small feature sizes. The experimental results show that the feature size of 55 gives the highest accuracy with an appropriate processing time.

We then extract packets with 55 features, and arrange the time series packets into a flow. Fig. 6 presents the impact of the time sequence length, i.e., the number of packets in a flow. This figure shows that the time sequence length 5 enhances the highest F1-score of the classifier as $98.17 \%$ in our network model. If we increase the time sequence length, we will have to enlarge the number of hidden layers in the deep network model to attain a high accuracy of classifiers as in the work [8].

Generally, an appropriate feature set can help the training process converge faster. Fig. 7 presents the convergence ability of our model with the input size of the feature set as $n \times 5 \times 55$, where $n$ is the time sequence length in the dataset. As shown in this figure, our network model converges quickly at 50 epochs. This conjectures that our feature set is appropriate to represent encrypted network applications for improving the performance of the application classification problem. Additionally, in the real network monitoring, if we choose the large number of packets presenting for a sequence, the model will only predict applications after passing many packets, leading to huge computation. Therefore, choosing sequence size of 5 is one of reasons that helps to rapid our model.

\section{B. Accuracy of the Model}

This section presents the effectiveness of our model on the dataset by comparing with other works in the same domain. Table II shows the Precision, Recall, and F1-score calculated on each application class. As shown in this Table, both unencrypted applications and encrypted applications are identified accurately with the average F1-score up to 0.98 . This
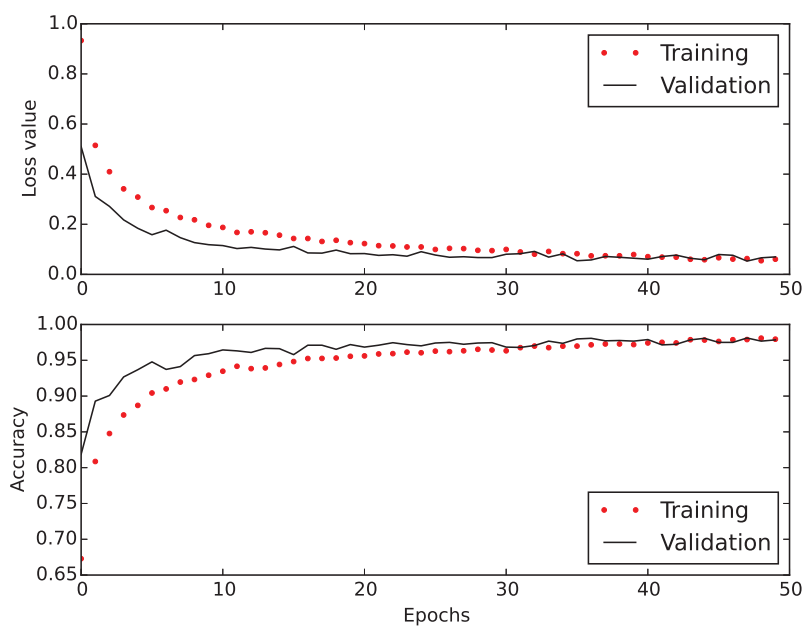

Fig. 7: Loss value and Accuracy in training process.

TABLE II: Accuracy on each class

\begin{tabular}{||c|c|c|c|c||}
\hline Class & Precision & Recall & F1 score & Sample size \\
\hline \hline Chat & 0.94 & 0.99 & 0.96 & 270 \\
Email & 0.99 & 1.00 & 0.99 & 76 \\
File transfer & 0.99 & 0.88 & 0.93 & 195 \\
P2P & 1.00 & 0.99 & 0.99 & 482 \\
Streaming & 0.99 & 1.00 & 0.99 & 563 \\
VoIP & 0.86 & 0.90 & 0.88 & 112 \\
VPN-Chat & 0.99 & 1.00 & 0.99 & 476 \\
VPN-Email & 1.00 & 0.98 & 0.99 & 91 \\
VPN-File transfer & 0.98 & 0.99 & 0.99 & 304 \\
VPN-P2P & 0.98 & 0.92 & 0.95 & 50 \\
VPN-Streaming & 1.00 & 0.99 & 1.00 & 155 \\
VPN-VoIP & 1.00 & 1.00 & 1.00 & 74 \\
\hline \hline Average & $\mathbf{0 . 9 8}$ & $\mathbf{0 . 9 8}$ & $\mathbf{0 . 9 8}$ & $\mathbf{2 8 4 8}$ \\
\hline
\end{tabular}

demonstrates that our deep network model and feature engineering technique are very efficiency in identifying encrypted network applications.

Finally, we compare our proposed solution with some previous works in the same domain which extract the payload of packets as features. We then use deep learning model to classify network applications. We experiment the model proposed by Martin et al. [8] on VPN-nonVPN dataset to compare performance reliability. In Table III, the network architecture shows the number of hidden layer and the the number of neurons in each hidden layers of models. For example, the model [8] uses the deep network with 5 hidden layers, i.e., two convolutional layers, one LSTM layer, and two FC layers, with the number of filters (for CNN layer) or neurons (for LSTM and FC layers) at each layer as 32, 100, 100, 100, and 108 respectively. Furthermore, M. Lotfollahi et al. [4] designed two deep network models, i.e., SAE and CNN1D with the accuracy as 0.97 on VPN-nonVPN dataset. As shown in this Table, our feature engineering technique combining with LSTM model achieves the highest accuracy of 0.98 with less complicated deep learning architecture which has only three layers, i.e., LSTM (128), FC (128), and FC (64). This verifies that our model is the most effective model for the encrypted network traffic classification problem in terms 
TABLE III: Comparision of deep learning methods based on packet payload features

\begin{tabular}{||c|c|c|c|c||}
\hline Network & $\begin{array}{c}\text { Feature } \\
\text { size }\end{array}$ & $\begin{array}{c}\text { Time } \\
\text { sequence } \\
\text { length }\end{array}$ & $\begin{array}{c}\text { Network } \\
\text { Architecture }\end{array}$ & $\begin{array}{c}\text { F1 } \\
\text { score }\end{array}$ \\
\hline \hline CNN+LSTM [8] & 1000 & 20 & $\begin{array}{c}\text { CONV.(32), } \\
\text { CONV.(100), } \\
\text { LSTM(100), } \\
\text { FC (100), FC(108) }\end{array}$ & 0.96 \\
\hline CNN-1D [4] & 1500 & 1 & $\begin{array}{c}\text { CONV.(200), } \\
\text { CONV.(100), } \\
\text { FC(600), FC(400), } \\
\text { FC(300), FC(200), } \\
\text { FC(100), FC(50) }\end{array}$ & 0.97 \\
\hline SAE [4] & 1500 & 1 & $\begin{array}{c}\text { FC(400), FC(300), } \\
\text { FC(200), FC(100), } \\
\text { FC(50) }\end{array}$ & 0.97 \\
\hline Our work & $\mathbf{5 5}$ & $\mathbf{5}$ & $\begin{array}{c}\text { LSTM(128), } \\
\text { FC(128),FC(64) }\end{array}$ & $\mathbf{0 . 9 8}$ \\
\hline
\end{tabular}

of computation resource and accuracy.

\section{SUMMARY}

In this paper, we propose a novel time series analysis for network traffic classification in order to effectively represent encrypted network applications. In particular, we first exploit significant features of network packets, then represent the flow of packets as the time series data. After that, we reconfigure data samples from raw packets to time sequence samples which can represent the behavior of network traffic. Furthermore, we take the advantage of LSTM network to design the deep network model which can learn feature effectively from time series data samples. The experimental results clearly show that using time series analysis of encrypted network traffic to represent encrypted network application combining with LSTM network can help the classifier achieve better performance for the encrypted network traffic classification. Our work can establish fundamental principle for utilizing time series features of encrypted network traffic and the deep learning approach to represent network traffic. This is the most important step in analyzing network traffic in order to provide valuable performance for various research works on network traffic analysis such as intrusion detection, anomaly detection, traffic classification.

\section{REFERENCES}

[1] T. T. T. Nguyen, and G. Armitage. "A survey of techniques for Internet traffic classification using machine learning," IEEE Communications Surveys \& Tutorials, vol. 10, no. 4, pp. 56-76, Sept. 2008.

[2] F. Zhang, W. He, X. Liu, and P. G. Bridges,"Inferring Users Online Activities Through Traffic Analysis," in ACM Conference on Security and Privacy in Wireless and Mobile Networks, Germany, Jun. 2011.

[3] P. Velan, M. Cermak, P. Celeda, and M. Draar. "A survey of methods for encrypted traffic classification and analysis," International Journal of Network Management, vol. 25, pp. 355-374, Jul. 2015.

[4] M. Lotfollahi, R. S. H. Zade, M. J. Siavoshani, and M. Saberian. "Deep packet: A novel approach for encrypted traffic classification using deep learning," arXiv preprint arXiv:1709.02656. Sept. 2017.

[5] A. Gharib, I. Sharafaldin, A. H. Lashkari, and A. A. Ghorbani. "An evaluation framework for intrusion detection dataset," International Conference on Information Science and Security, pp. 1-6, Thailand 2016.

[6] I. Sharafaldin, A. H. Laskari, and A. A. Armitage "Toward generating a new intrusion detection dataset ans intrusion traffic characterization," International Conference on Information Systems Security and Privacy, Purtogal, Jan. 2018.

[7] R. Alshammari and A. N. Z. Heywood, "Can encrypted traffic be identified without port numbers, IP addresses and payload inspection?," Elsevier, vol. 22, pp. 1326-1348, ISBN 1326-1350, 2010.

[8] M. L. Martin, A. S. Escuevillas, and J. Lloret. "Network traffic classifier with convolutional and recurrent neural networks for internet of things," IEEE Access, vol. 5, no. 18042-18050, 2017.

[9] N. Moustafa and J. Slay, "UNSW-NB15: A comprehensive data set for network intrusion detection systems (UNSW-NB15 network data set)," Military Communications and Information Systems Conference, 2015.

[10] http://nsl.cs.unb.ca/NSL-KDD/

[11] M. Finsterbusch, C. Richter, E. Rocha, J. A. Muller, and K. Hanssgen, "A Survey of payload-based traffic classification approaches," IEEE Communications Surveys and Tutorials, vol. 16, no. 2, pp. 1135-1156, Aug. 2014.

[12] D. Nayor, A. Finamore, I. Leontiadis, Y. Grunenberger, M. Mellia, M. Munafo, K. Papagiannaki, and P. Steenkiste, "The cost of the S in HTTPS," CoNEXT '14 Proceedings of the 10th ACM International on Conference on emerging Networking Experiments and Technologies , pp. 133-140, Sydney, Australia, Dec. 2014.

[13] http://www.unb.ca/cic/datasets/vpn.html

[14] G. D. Gerard, A. H. Lashkari, M. Mamun, and A. A. Ghorbani. "Characterization of encrypted and VPN traffic using time-related features," International Conference on Information Systems Security and Privacy, 2016.

[15] J. Sherry, C. Lan, R. A. Popa, and S. Ratnasamy, "Blindbox: Deep packet inspection over encrypted traffic," ACM SIGCOMM Computer Communication Review, 45(4), pp.213-226, 2015.

[16] S. Leroux, S. Bohez, P. J. Maenhaut, N. Meheus, P. Simoens, and B. Dhoedt. "Fingerprinting encrypted network traffic types using machine learning," NOMS2018, the IEEE/IFIP Network Operations and Management Symposium,(pp. 1-5), 2018.

[17] Y. Bengio, P. Simard, and P. Frasconi. "Learning long-term dependencies with gradient descent is difficult," IEEE Transactions Neural Networks, pp.157-166, 1994.

[18] S. Hochreiter and J. Schmidhuber. "Long Short-Term Memory," Journal Neural Computation, vol. 9, no. 8, pp.1735-1780, Nov. 1997

[19] Z. C. Lipton, J. Berkowitz, and C. Elkan. "A Critical Review of Recurrent Neural Networks for Sequence Learning," Neural and Evolutionary Computing, May 2015.

[20] N. Singhal and J. P. S. Raina. "Comparative Analysis of AES and RC4 Algorithms for Better Utilization" in International Journal of Computer Trends and Technology, ISSN: 2231-280, July to Aug Issue 2011, pp. 177-181.

[21] D. M. W. Powers, "Evaluation: from precision, recall and F-measure to ROC, informedness, markedness and correlation," Journal of Machine learning Technologies, vol. 2, pp. 37-63, 2011. 